\begin{tabular}{|l|l|l|l|l|l|}
\hline Revista Clío América & ISSN: 1909-941X & Vol. 10 & No. 20 & Julio - Diciembre de 2016 & 194 - 211 \\
\hline \multicolumn{6}{|c|}{ DOI: http://dx.doi.org/10.21676/23897848.1877 } \\
\hline
\end{tabular}

\title{
Inteligencia de los negocios \\ Clave del éxito en la era de la información
}

\author{
Business intelligence \\ Key to success in the information age
}

Resumen: Este artículo tiene por objetivo describir y clasificar de una forma más concreta los sistemas de información, los cuales se encuentran enmarcados en lo que se denomina propiamente como "la inteligencia de los negocios". Se inicia analizando cada uno de los conceptos básicos y aspectos teóricos, para luego detallar en la conformación de los sistemas transaccionales y los sistemas estratégicos más relevantes incluyendo sus beneficios, sus modos de uso, y sus desventajas; se reflexiona sobre algunos casos expuestos de la realidad empresarial a nivel global, y el impacto que ha tenido la adopción de los sistemas de información en su estrategia corporativa como clave del éxito logrando ventajas competitivas considerables.

Finalmente se concluye describiendo la relevancia que ha tenido la implementación de por lo menos alguno de estos sistemas de información en la planeación estratégica de las organizaciones, especialmente en Colombia y Latinoamérica.

Palabras clave: Inteligencia de los negocios, sistemas de información, sistemas transaccionales, sistemas estratégicos.

JEL: 03

\begin{abstract}
This article aims to describe and classify in a more concrete way information systems, which are framed in what is properly called as "business intelligence". It begins analyzing each of the basic concepts and theoretical aspects and then detail in shaping transactional systems and the most important strategic systems including their benefits, their modes of use, and its disadvantages; It reflects on some cases actually exposed business globally, and the impact it has had the adoption of information systems in its corporate strategy as a key competitive advantages achieving considerable success.
\end{abstract}

Finally, it concludes by describing the importance that has had the implementation of at least some of these information systems in strategic planning of organizations, especially in Colombia and Latin America.

Keywords: Business intelligence, information systems, transaction systems, strategic systems.

\section{Ind}

Helmer Muñoz-Hernández

Ingeniero de Sistemas de Información,

Magister en Ingeniería de Control

Industrial, Doctor en Ciencia y Tecnologìa,

Docente investigador de la Corporación

Unificada Nacional de Educación

Superior-CUN, Grupo de investigación

AXON-Colombia.

Email: helmer_munoz@cun.edu.co

Roberto Carlos Osorio-Mass

Ingeniero Industrial, MBA en Dirección y

Administración de Empresas. Esp. Gerencia

de Mercadeo, Docente Investigador de la Corporación Unificada Nacional de Educación

Superior-CUN, Grupo de investigación GIDECER-Colombia.

Email: roberto_osoriom@cun.edu.co

Luis Manuel Zúñiga-Pérez

Administrador de Empresas, Especialista en Docencia Universitaria, Docente Investigador de la Corporación Unificada Nacional de Educación Superior-CUN , Grupo de investigación GIDECER-Colombia. Email: luis_zuniga@cun.edu.co

Tipología:

Artículo de Revisión

Fecha de Recibido:

Abril 25 de 2016

Fecha de Aceptación:

Agosto 08 de 2016

Para citar este artículo:

Muñoz, H. H., Osorio, M. R., \& Zúñiga, P.L. (2016). Inteligencia de los negocios. Clave del Éxito en la era de la información. Clío América, 10 (20), p.p. 194 - 211 


\section{Introducción}

\section{Inteligencia de los negocios}

Se puede decir que son aquellos recursos administrativos empresariales con los que las organizaciones actuales y modernas pueden contar para aprovechar al máximo toda la información que posean tanto de sus clientes como la de sus proveedores y hasta la de sus competidores inclusive; todo con el fin de lograr ventajas competitivas en un mercado hostil y demasiado dinámico.

No obstante, en el actual entorno de revolución tecnológica y abundante información, las organizaciones tienen que intensificar sus estrategias en función de integrar grandes cantidades datos dispersos.

El manejo de la administración, la gestión y control de la información como un arma estratégica, forma parte de la inteligencia del negocio, con apoyo de herramientas informáticas y analíticas que ayudan a las organizaciones a maximizar su rendimiento generando eficacia operativa. Así mismo, la gestión del conocimiento ayuda a obtener mayor comprensión y entendimiento del entorno y de los procesos desde la propia experiencia de las personas y organizaciones. (Dávila L. F., 2005, p.17)

Existen muchas definiciones de inteligencia de negocios (BI), sin embargo, se rescatan dentro de las más integrales:

Hatch (citado por Rodriguez, 2014) define que:

Business Intelligence (BI) es la combinación de prácticas, capacidades y tecnologías usadas por las compañías para recopilar e integrar la información, aplicar reglas del negocio y asegurar la visibilidad de la información en función de una mejor comprensión del mismo $y$, en última instancia, para mejorar el desempeño. (p. 103).

Para Médes (2006) "el término de Business Intelligence se reconoce como el valor de suministrar hecho e información como soporte a la toma de decisiones" (p.21). Así mismo concluye en ralacion a la inteligecia del negocio como:
El conjunto de herramientas y aplicaciones para la ayuda a la toma de decisiones que posibilitan acceso interactivo, análisis y multiplicación de la información corporativa de misión crítica. Estas aplicaciones aportan un conocimiento valioso sobre la información operativa identificando problemas y oportunidades de negocio. Con ellas los usuarios son capaces de acceder a grandes cantidades de información para establecer y analizar relaciones y comprender tendencias que, a la postre, soportaran decisiones de negocio. (p. 23,24).

Pero no se debe confundir el concepto de inteligencia de negocio con el almacenamiento de grandes datos de información; $B I$ es el puente para que las empresas puedan hacer útil dicha información mediante herramientas puestas al servicio de os usuarios (Howson, 2010, p.2).

Hay elementos que están muy correlacionados con la inteligencia de los negocios, por cuanto serían los factores claves en la consecución de lo que más adelante detallaremos como los procesos de negocio. Son estos elementos, el conocimiento, los datos y la información; los datos se transforman en información, y esta a su vez en conocimiento; y esto es BI. Por tanto:

Los datos son la mínima unidad semántica, y se corresponden con elementos primarios de información que por sí solos son irrelevantes como apoyo a la toma de decisiones. También se pueden ver como un conjunto discreto de valores, que no dicen nada sobre el porqué de las cosas y no son orientativos para la acción.

La información se puede definir como un conjunto de datos procesados y que tienen un significado (relevancia, propósito y contexto), y que por lo tanto son de utilidad para quién debe tomar decisiones, al disminuir su incertidumbre.

El conocimiento es una mezcla de experiencia, valores, información y know-how que sirve como marco para la incorporación de nuevas experiencias e información, y es útil para la acción. Se origina y aplica en la mente de los conocedores. (Davenport \& Prusak, L., 1999). 


\section{Figura 1.}

\section{Conocimiento, información, y datos}

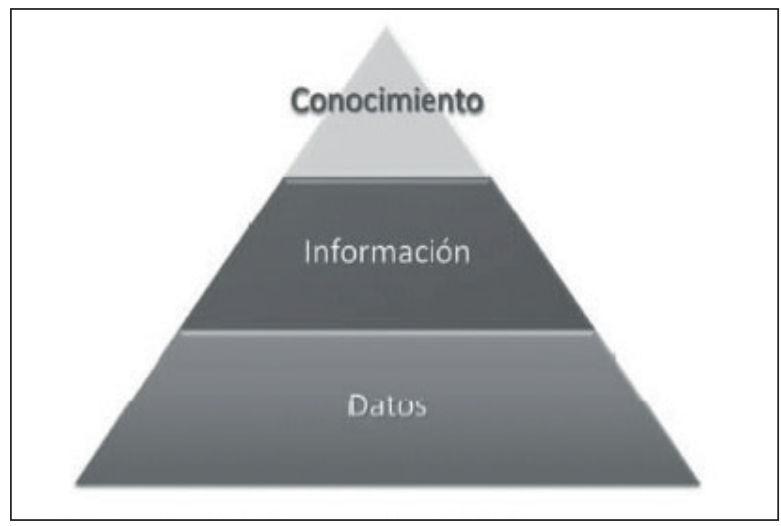

Fuente: (Davenport \& Prusak, 1997).

\section{¿Cómo funciona La BI en las áreas de la empresa?}

La inteligencia del negocio como ya se sabe es el eje integrador de la información en la empresa, por tanto, son las propias áreas ésta el foco de acción de la BI.

Se dice que cada departamento de la organización acumula múltiples datos pertenecientes a su propia actividad en la empresa; sin embargo, las oportaciones a cada departamento pueden ser notorias. Rojas, G, (2016) desglosa asi:

- En Marketing, la BI permite identificar de forma más precisa los segmentos de clientes y estudiar con mayor detalle su comportamiento.

- En compras, permite acceder a los datos del mercado, vinculándolos con la información básica necesaria para hallar las relaciones entre coste y beneficio.

- En producción, proporciona un mecanismo que permite analizar el rendimiento de cualquier tipo de proceso operativo, ya que comprende desde el control de calidad y la administración de inventarios hasta la planificación y la historización de la producción.

- En ventas, facilita la comprensión de las necesidades del cliente, así como responder a las nuevas oportunidades del mercado. (p.46, 47)

La inteligencia de los negocios y su aplicabilidad en los mismos sirven de soporte especial, a la organización para los procesos y operaciones, e indudablemente para la toma de decisiones; este apoyo que le otorga la BI a las organizaciones se da de manera organizada y estructurada, y se logra teniendo en cuenta tres frentes fundamentales en las cuales la BI se desarrolla: a nivel estratégico, táctico y operativo como se muestra en la figura 2 (Zarate, 2013).

De igual forma para O'Brien (2006) "estos niveles son: los de apoyo en los procesos y operaciones; los de apoyo en la toma de decisiones, y los de apoyo estratégico para lograr ventajas competitivas" (p. 8).

Apoyo a los procesos de negocio o a nivel operativo; por ejemplo, los negocios utilizan los sistemas de información para ayudarse a registrar las compras de los clientes, tener al día el inventario, la nómina, las compras, y la evaluación de nuevas tendencias.

Apoyo en la toma de decisiones o a nivel táctico; los sistemas de información ayudan a los gerentes y a los profesionales de los negocios a tomar decisiones. Por ejemplo, decisiones acerca de que líneas de productos lanzar o retirar del portafolio, o que tipo de inversión requieren, son realizadas por los sistemas de información.

Apoyo en la ventaja competitiva o a nivel estratégico; lograr una ventaja competitiva sobre los competidores, requiere de una aplicación innovadora de las tecnologías de información. Por ejemplo, la dirección de una cadena de tiendas decide instalar estaciones con pantallas digitales en todas sus sucursales con vínculos en su sitio Web de comercio electrónico para las compras en línea. Esto podría atraer nuevos clientes, creando lealtad de os mismos debido a la comodidad de ir a las tiendas y comprar mercancía suministrada por los sistemas de información. Por eso los sistemas de información pueden ayudar a proveer productos y servicios que dan a un negocio una ventaja competitiva sobre sus competidores. (0'Brien, 2006, p. 8).

Conforme a lo anterior, son esas aplicaciones tecnológicas como el CRM, SCM, BSC, ERP, y el EIS, los principales sistemas de información que se abordaran más adelante y su impacto en los procesos de las organizaciones. 


\section{Figura 2.}

\section{Niveles de orden en función de la inteligencia de negocios}

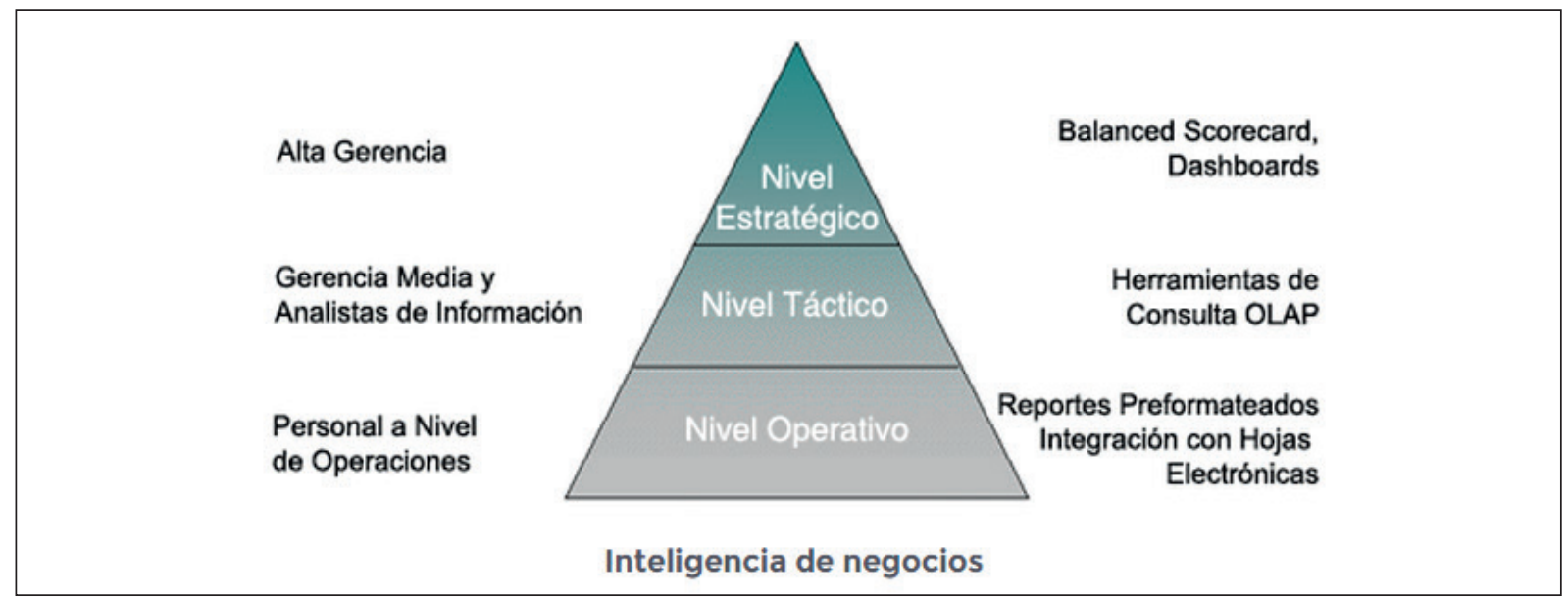

Fuente: (Zarate, 2013)

\section{Antecedentes}

Hablar de Business inteligence (BI), no es relativamente nuevo, los primeros conceptos surgen con la aparición de un sistema llamado "avant la lettre". Cobarsí, (2011) describe:

Se trata del sistema de defensa aérea de Inglaterra creado en la década de 1930 y utilizado durante la Segunda Guerra Mundial, principalmente en el año 1940.

En los años treinta del siglo XX, los responsables técnicos del diseño de la defensa aérea británica se planteaban cómo defender Inglaterra de un posible ataque aéreo a gran escala.

La primera alternativa que se exploró fue un nuevo «supercañón» antiaéreo, capaz de lanzar «rayos de la muerte» a gran distancia contra los aviones enemigos. Pero una vez estudiada su viabilidad tecnológica se descartó. En cambio se consideró factible utilizar un emisor de rayos de ondas electromagnéticas (es decir, el ingenio que ahora denominamos radar) para detectar desde lejos los aviones enemigos, prever la trayectoria e interceptarlos con cazas propios. Esto permitió a los británicos construir un sistema de información que contribuyó no- tablemente a evitar una invasión terrestre de las Islas Británicas. (p.11, 12).

Hacia los años 60's las empresas solo se apoyaban en los departamentos de sistemas, los cuales hacían los reportes a través de grandes computadoras centralizadas con grandes bases de datos. Con la explosión de las computadoras para los años 70's millones de usuarios fueron adoptando el uso de estos equipos y cobrando fuerza hasta convertirse en moda (Zarate, 2013).

Para los años $80^{\prime}$ s y los $90^{\prime}$ s, se empezaron a gestionar grandes bases de datos con lo que permitía analizar grandes cantidades de información en un tiempo muy corto y real. En el nuevo milenio se da lo que llamaremos el CRM (Customer Relationship Management), concepto que detallaremos más adelante. Finalmente, para el 2005 entra el término objeto de estudio de este artículo, Business Intelligence (BI).

Actualmente se puede acceder al cliente de diversas formas, puede que sea por medio de un mail, una video llamada, por medio de una página web, inclusive por medio de mensajes de texto a través del móvil, y más aún por medio de las redes sociales que ha sido una de las formas más estratégicas de interacción de los clientes con la empresa. 
Sin embargo, también es muy común que se pueda ir al cine o comprar un tiquete de avión desde la casa a través de internet, incluso descargar el código QR desde los Smartphone; por lo que se puede intuir que la conveniencia en estos casos es muy alta. Es precisamente en este momento donde el cliente 0 usuario elige el canal que prefiere utilizar en cada instante al momento de la compra.

Resulta interesante y conveniente imaginar una situación comercial en el que, por ejemplo, un cine conozca los gustos y preferencias de sus clientes y que por medio de un SMS de un aviso del último estreno de su interés, y que este aviso contenga un link que lo traslade a un sitio web donde pueda hacer su reserva o su compra directa. Esta y muchas más conveniencias son las resultantes de lo que es la (BI).

\section{Beneficios.}

Herrera, (2015) describe los beneficios que se pueden adquirir a través de la BI son diversos:

- Beneficios tangibles: se logra reducción de costes, generación de ingresos y reducción de tiempos en varias actividades.

- Beneficios intangibles: en referencia a que más usuarios harán uso de la información en la medida que esté disponible para la toma de decisiones.

- Beneficios estratégicos: hacen referencia a decisiones de alto nivel, como política de mercados, productos etc. (Cano, 2007)

\section{El uso}

Para los directores de empresas, gerentes y cargos similares la aportacion que hace la BI es crucial; se podrian imaginar profesionales en estos cargos sin hacer seguimeinto a las ventas, a los costes y gastos generados, o peor aun, sin hacer proyecciones por falta de información historica. De ahí la importancia de la BI y los sistemas de información que las organizaciones adopten para el desarrollo de sus actividades y gestiones empresariales (Polo, A, 2009).

\section{Los Sistemas de información}

Los procesos de negocio son todas las actividades interdepartamentales y correlacionadas entre sí, para el funcionamiento lógico de una empresa. Ejemplos claros: el proceso de producción, la nómina, la logística de entrada, la de salida, reclutamiento de personal etc.

Ahora bien, todo proceso está asociado a un intercambio de información la cual es la función primordial de la gestión estratégica la información.

Los sistemas de información han contribuido a los negocios innovando nuevas formas de desarrollar los procesos; adoptando las nuevas tecnologías en un medio más digital; aportando a la trasformación de la empresa comercial; y contribuyendo a una economía más globalizada. (Rocha, 2007).

\section{Antecedentes.}

La existencia de los sistemas de información se remonta desde los principios de la historia de la humanidad; sin embargo, detallaremos algunos apartes importantes de su trayectoria como lo expresa Sosa \& Hernández, (2007) en su resumen historico:

Hasta el momento, los documentos más antiguos que se conocen fueron descubiertos en los años 3000- 2800 (a.n.e), cerca del antiguo estuario de Eufrates, en Mesopotamia. Se trata de libros de cuentas y de inventarios redactados en escrituras cuneiforme arcaniana en tabletas de arcillas. Paralelamente aparece en Egipto la escritura jeroglífica, destinada originalmente a inscripciones en monumentos y edificios.

En los años 79-78 (a.n.e) se constituye en Roma el Tabularium (archivos centrales del imperio romano), en el cual se almacenaban las tarjetas de madera blanqueada (álbumes) donde recogían los hechos económicos y sociales.

En el año 98 (d.n.e) fue la creación de tarjetas perforadas por el Dr. Herman Hollerith, estas 
máquinas proveyeron el soporte técnico para procesar grandes volúmenes de información estadística, contable y de gestión en general.

Con el pasar de los años el mecanismo de almacenar la información se fue desarrollando y se crearon equipos novedosos que facilitaron realizar disímiles operaciones. Se sucedieron unas tras otras las generaciones de computadoras, surgieron nuevos medios de almacenamiento, más capaces (p.6, 7).

\section{$\underline{\text { Características }}$}

Los sistemas de información plantean las siguientes características según Berenguer \& Ramos-Izquierdo, (2003) así:

- En una organización pueden existir varios sistemas de información.

- Algunos sistemas de información pueden ser totalmente independientes, pero la mayoría esta interconectados.

- Un sistema de información en términos generales se compone de hardware conectado mediante redes (Internet LAN o WAN), además de software, base de datos, datos y procedimientos de funcionamiento.

- Los sistemas de información pueden ser desarrollados a medida para la empresa, 0 adquiridos en el mercado como paquetes de software estándar. (p. 72)

Los procesos de negocios o sistemas de información, se dividen en dos tipos: los procesos transaccionales y los procesos estratégicos.

Los procesos transaccionales son aquellos que se encargan de gestionar la información de forma muy detallada. Por ejemplo; la parte contable de una empresa, el cual almacena y trata la información financiera, las ventas, la nómina etc. (Ver figura 3).

Ahora, los procesos estratégicos son los que se encargan de gestionar la información de forma más sumarizada y analizada, con la finalidad de interpretar y decidir a partir de los resultados obtenidos de las diversas operaciones claves de la empresa como las ventas, el gasto, los impuestos etc.
Figura 3.

\section{Tipos de Sistemas de Información}

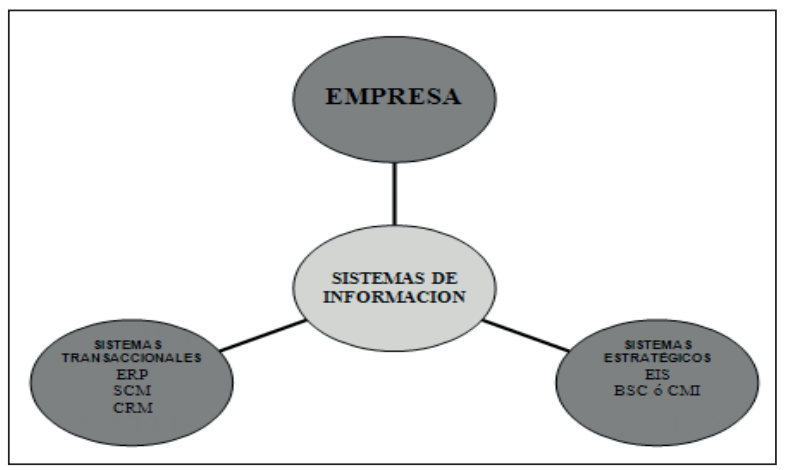

Fuente: Elaboración propia

\section{Los Sistemas Transaccionales}

"Los sistemas transaccionales son los sistemas enfocados en la toma de decisiones, que tienen como función la manipulación de la información, con el fin de apoyar y fundamentar la toma de decisiones". (Cohen \& Asín, 2014).

Sin embargo Marco \& Marco, 2013 afirma que: "Su objeto es la transacción (de aquí el segundo nombre de estos sistemas), es decir, el registro de todas las actividades y acciones particulares y formales de la organización con su entorno y dentro de sí misma" (p.215).

Dentro de los procesos transaccionales podemos encontrar el ERP, el SCM, y el CRM.

\section{La planificación de los recursos de la organización. (ERP).}

0 bien conocidos en inglés como "Enterprise Resource Planning". Este sistema administra y controla la información al interior de la empresa de forma muy detallada.

Cobarsí, (2011) afirma:

Estos sistemas permiten una gestión integrada de los datos de la empresa, sobre un solo repositorio, 
aúnan la gestión de procesos en los que intervienen diferentes departamentos, refuerzan el control sobre la estructura organizativa y fomentan una mayor disciplina en la organización. Permiten conseguir mejoras en aspectos como: control y reporting (informes de situación para la dirección), mayor rapidez de los procesos, reducción de inventarios, reducción del time to market, mejora del servicio a los clientes. Se aplican tanto a empresas industriales como de servicios. (p.62, 63).

Los ERP tienen un carácter generalizado en el sentido que puede incluir otros sistemas de información transaccional como el CRM y el SCM. Dentro de sus principales funciones del se encuentran la sincronización de las actividades internas de la empresa, la administración de la información, y la optimización de los recursos de la misma.

Este sistema de negocio puede contener varios módulos puesto comparten la misma base de datos; es así: la gestión de producción, gestión de clientes, compras, cuentas a pagar y cobrar, contabilidad general, facturación, gestión de inventario, recursos humanos, nóminas o cualquier otra función que se tenga que desarrollar dentro de la empresa. (Arjonilla \& Medina, 2013, p.122).

Figura 4.

\section{Valor de un negocio ERP}

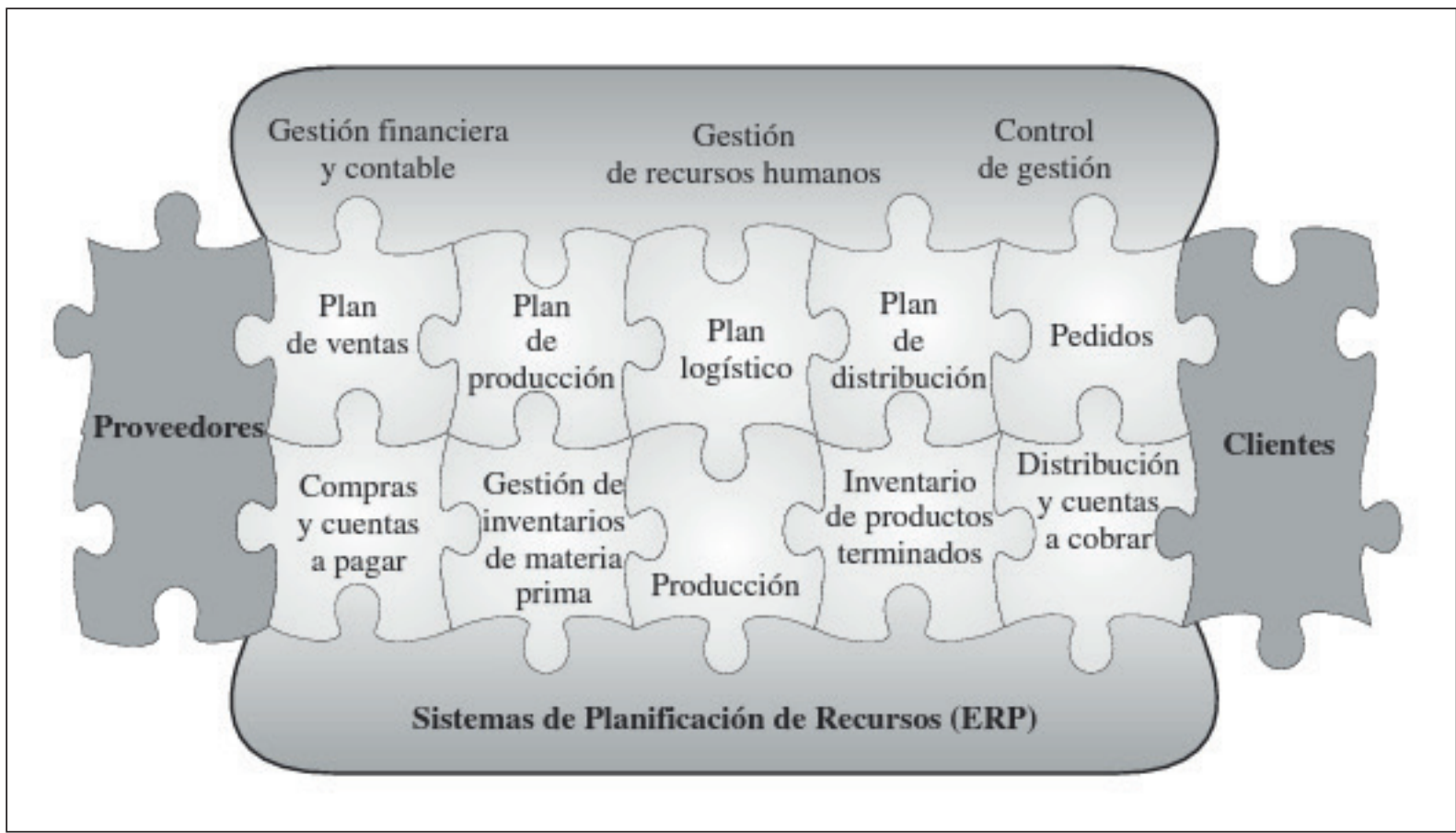

Fuente: (Arjonilla \& Medina, G, 2013.p.122)

Ahora bien, así como es atractivo integrar toda la información empresarial en un mismo paquete, lo que generaría valor para el negocio, es importante saber en qué momento la empresa necesita un sistema de negocios de este tipo, puesto que hay alertas en la empresa que describen la necesidad como, por ejemplo: departamentos que duplican trabajo, reproceso, falta de información para la toma de decisiones, entre otros; todos propios de empresas en etapa de crecimiento (Arjonilla \& Medina, 2013). Sin embargo según Sánchez, Toharia, \& Raya, 2000 "no todas las empresas se pueden definir con los 
modulos anteriormente mecionados. Por ello los sistemas ERP se complementa con otros modulos" son estos:

- Prevision de la demanda.

- Mantenimiento.

- Gestion de cabios del producto (PDM o Product Data Magnagement).

- Configuracion de productos a medida.

- Gestion de las relaciones con los clientes (CRM).

- Controles de planta y almacenes.

- Seguridad, planes de contnuidad y contingencia. (p.210)

Los principales retos o desafíos que enfrenta un sistema de negocios como estos, están plasmados en diversos tipos: en lo económico, por cuanto muchas empresas no pueden soportar un gasto de tal magnitud; en lo cultural, es difícil el cambio, romper con las practicas rutinarias y los viejos paradigmas más aún cuando la empresa no es flexible y busque una mejor adaptación; y en la implementación, puesto que el proceso surgen defectos y habrá personas que se adaptan más fácil y logran entender rápidamente los cambios, e interactúan más eficientemente; esto conlleva a sentirse más importantes que otras, y niegan en compartir la información con el resto de personal, o lo hocen de forma parcial (Mozqueda, 2016).

\section{Ventajas y beneficios}

Se podrían mencionar algunas de las ventajas y beneficios de un ERP de última generación según Cohen \& Asín, 2009:

- No solo apoyan y optimizan los recursos de una empresa, sino comparten la información con empresas vinculadas.

- Los nuevos ERP amplían sus funciones, incluso aquellas más específicas de industria individuales.

- Tecnologías se basan en protocolos de comunicación de internet

- -Un ERP ofrece mejores prácticas a la industria, así la empresa estará a niveles equivalentes a la competencia.

- Al tener todos los sistemas de los diferentes departamentos integrados se logra una visión global de la operación de la empresa.
- Los procesos de la cadena de suministro y producción, al estar automatizados, harán que la empresa tenga flexibilidad y agilidad en su operación y mejoran sus niveles de inventario.

- Los procesos de atención al cliente mejoraran debido a la posibilidad de tener información relevante de los eventos que vinculan al cliente con la empresa. (p.102).

Así mismo, algunas características particulares de este sistema estan descritas en acciones propias de un ERP como es la integración de los procesos, adaptación a los mismos, interactividad, flexible en la utilización a nivel mundial (Fernández \& Navarro, 2014).

\section{Un caso de éxito de ERP: Walmart.}

La clave del éxito de esta gran compañía se debe a su excelente operación logística por lo que ha sabido manejar muy bien sistemas estratégicos de información como el ERP. Walmart integró un sistema de información universal y lo expandió a todos sus colaboradores estratégicos, proveedores e intermediarios para que conocieran en tiempo real cómo estaban rotando sus mercancías en los puntos de ventas de la cadena de cada país, de cada ciudad. De ésta manera el proveedor o los suministradores podían conocer y planificar el próximo aprovisionamiento sin causar desabastos en el supermercado e inconvenientes con el cliente.

Pero para lograr esta sincronización efectiva en el suministro, Walmart disponía dentro de sus políticas internas, pactar dentro de los contratos con sus proveedores, el compromiso de que éstos mismos por obligación tenían que integrar en sus operaciones, sistemas de ERP para que se pueda hacer las lecturas en tiempo real de las necesidades que se generaban en el punto. Actualmente, Walmart mantiene su estrategia adaptándola cada día más a las exigencias de los consumidores y que a pesar de compartir información crucial de sus operaciones con sus aliados estratégicos, queda por sentando que no siempre los secretos son las claves del éxito.

La administración de la cadena de suministros (SCM)

También conocido como Supply Chain Management y consiste en la administración eficaz en términos de 
información, de la cadena de suministro, haciéndola extensiva hacia los proveedores y finalmente a los clientes finales.

Se caracteriza por administrar controla el aprovisionamiento de materias primas, la planificación de la producción y por último en control y distribución del producto terminado.

No obstante, es preciso rescatar conceptos muy ligados a la cadena de suministro; uno de ellos es la logística.

Velazco (2013) describe que:

La logistica es la parte del proceso de la cadena de suministro que planea, Lleva a cabo y controla el flujo y almacenamiento eficiente y efectivo de bienes y servicios, asi como de la informacion relacionada desde el punto de origen hasta el punto de consumo, con el fin de satisfacer los requerimientos de los clientes. (p.30)

Existen varios autores que definen a la cadena de suministros de forma muy concreta y con mucho contexto; sin embargo, para Pires \& Carretero, 2007:

Una cadena de suministros se define como el conjunto de todos los procesos que involucran a los proveedores y sus clientes y conectan empresas desde la fuente inicial de materia prima hasta el punto de consumo del producto acabado; las funciones dentro y fuera de una empresa que garantizan que la cadena de valor pueda elaborar y proveer de productos y servicios a sus clientes. (p.23).

Figura 5.

\section{Representación de la de la cadena de suministro SCM}

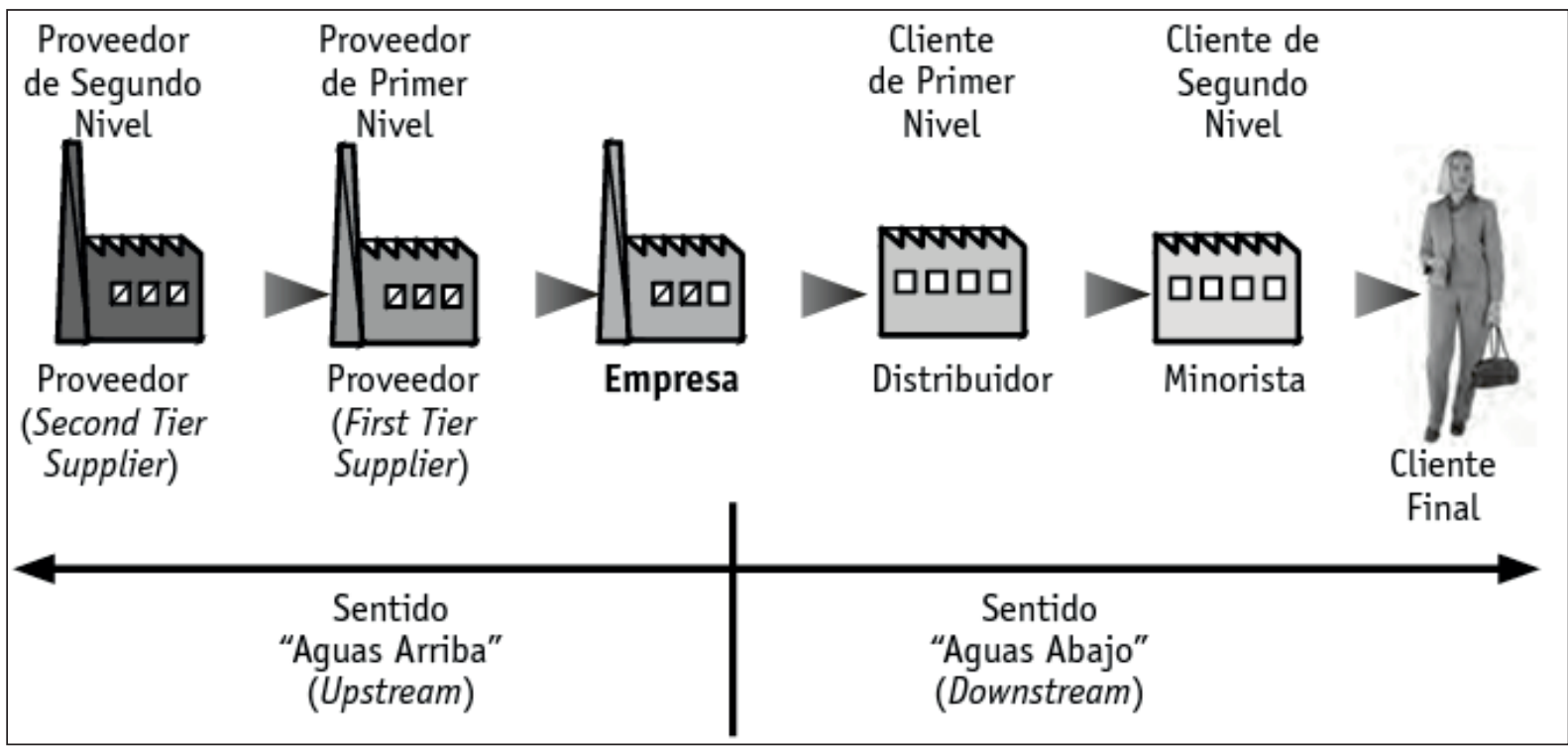

Fuente: (Pires \& Carretero, D, 2007. p.24)

La gestión de la cadena de suministro es una actividad multifuncional en la cual abarca áreas de actuación muy importantes al interior de la empresa; estas áreas son las que dan el origen a dicha cadena e impactan definitivamente el actuar de la SCM en la organización; por tanto, son estas áreas fundamentales la producción, la gestión de compras, la logística, y la parte comercial (Pires \& Carretero, 2007, p. 36). 
Asi mismo, Chavez, (2000) sostiene que la cadena de suministros: "Es un conjunto de actividades de abastecimiento, transformación y transporte enlazadas con actividades similares de los proveedores y clientes" (p.20).

Sin embargo, Christopher, 1998 describe la cadena de suministros como:

Una red de organizaciones que están relacionadas a través de las conexiones downstream 0 corriente abajo (en la dirección de creación de un producto o servicio) y upstream o corriente arriba (en la dirección de los proveedores) en los diferentes procesos y actividades que producen valor en la forma de productos y servicios finales ofrecidos al consumidor final. (p.24)

Esta herramienta es muy útil para empresas muy grandes con operaciones de abastecimiento muy frecuentes, como la de productos de consumo masivo ya que permite optimizar niveles de inventario al tiempo que los vuelve más transparentes; permite observar la rotación real de los productos en diferentes estacionalidades de tiempo, permite establecer tiempos de entrega, también permite establecer el coste de transporte de ciertos productos y así plantear estrategias de comercialización; finalmente permite hacer depuraciones en el portafolio de productos en pro de evitar pérdidas por obsolescencia.

Pero quizás la característica más importante de este tipo de sistemas de información, es la colaboración entre las dependencias, incluso entre empresas colaboradoras proveedores, distribuidores y demás; esto contempla ser una herramienta muy integradora con la cual las responsabilidades son compartidas y no dependen de una sola área; por tanto si un eslabón falla pierde toda la operación.

\section{Principios de la SCM}

En función de la gestión empresarial, la SCM contempla los siguientes principios básicos. Se especifican los siguientes Según Portal, (2011)

- Segmentar a los clientes de servicios y adaptar la cadena de acuerdo a esto.
- Ajustar la red de logística a los requerimientos de servicio y a la rentabilidad de los segmentos de cliente.

- Permanecer al asecho de las señales del mercado, la evolución tecnológica y de comportamiento del consumidor.

- Administración estratégica de las fuentes de suministro e insumos, con beneficios de mutua conveniencia.

- Desarrollar una estrategia tecnológica para toda la cadena de suministros.

- Crear mediciones del desempeño para todos los eslabones de la cadena de suministro con criterios de realidad, análisis y ajuste.

- Actualizar permanentemente todas las herramientas (técnicas, producción, administrativas y mercadológicas), para optimizar el proceso. (p.20)

\section{Beneficios del SCM}

Así mismo, la adopción de estos procesos de negocio, contempla beneficios muy importantes para la organización como destacan varios expertos:

- Reducción del stock; cuando se hace una adecuada práctica en el ejercicio de la SCM, acertando en los pasos de planificación de la producción (SNP) y la panificación de demanda (DP) (Christopher, 1998).

- Una reducción en costos asociados; en cuanto más ajustado estén los niveles de inventario menor será su coste de mantenimiento (Lambert \& Cooper, 2000).

- Un horizonte temporal de largo plazo: se asientan lazos de confianza entre los miembros de la cadena de suministro, como su coordinación y contratos menos detallados (Cavinato, 1991).

- Una disminución del tiempo del ciclo del producto; se reduce significativamente gracias a los esfuerzos en el buen manejo de las operaciones logísticas y prácticas en el control del stock (Cooper \& Ellram, 1993).

\section{Un caso de éxito de SCM: Bimbo}

Como recordaremos habrá muchas empresas que hacen de esta herramienta su factor clave del éxito; 
no obstante, hay una muy especial, y es el caso de la empresa Bimbo.

Velásquez, (2013) establece en relacion a este caso de éxito.

Esta gran compañía creó una nueva área funcional de SCM con el objetivo de unificar y optimizar la cadena de suministro y garantizar la máxima frescura de los productos, el mejor servicio al cliente y la rentabilidad de la gestión logística e industrial. La empresa necesitaba una herramienta para planificar la cadena de suministro de los productos de vida media y larga (pastelería, tostados y aperitivos y golosinas).

Para ello, el área de SCM procedió a realizar un estudio funcional y organizativo con el objetivo de optimizar la cadena de suministro, conseguir a la vez un frescor óptimo de los productos, disminuir los stocks intermedios en la cadena, optimizar la producción y mejorar el servicio a las delegaciones de venta desde las fábricas. (p.7)

Se puede decir que Bimbo integra una adecuada implementación de software específico (SAP), como plataforma para la herramienta SCM, sincronizando y planificando sus componentes básicos: planeación de la demanda (DP), planificación de la producción (SNP), y la gestión de aprovisionamiento logístico.

En conclusión, el SCM es una herramienta optimizadora que puede llegar a representar la clave del éxito en desarrollo de las operaciones de las organizaciones; no obstante, existe un concepto errado de que el SCM, es solo simplemente una cadena que interactúa con base a una plataforma tecnológica. En realidad, la importancia de la gestión de la cadena de suministros radica en las "personas" quienes, a través de sus acciones y su pro-actividad, dinamizan la cadena y la hacen una herramienta eficaz.

Surgen entonces conceptos relacionados como la supply chains dinámicas, haciendo referencia más a la gestión orgánica que lo brindado por la tecnología (Gattorna, 2011).

\section{CRM (Customer Relationship Management)}

Quizás sea uno de los procesos de negocios con mayor impacto dentro de las organizaciones al momento de adoptar nuevas estrategias que den un valor diferenciador en el mercado.

Muchas definiciones concretas y complejas de este sistema de negocio existen, se pueden rescatar algunas:

Ayuso \& Rodríguez, (2011) afirma que:

El CRM (Customer Relationship Management) hace referencia tanto a la estrategia de negocio, enfocada a seleccionar y gestionar una relación con los mejores clientes para optimizar su valor a largo plazo, como a las aplicaciones concretas de software necesarias para procesar la información de esos clientes y desarrollar esa relación. Es frecuente el uso de los términos CRM y marketing relacional como sinónimos, e incluso hablar de CRM para referirse a la estrategia de marketing de una compañía claramente orientada a la creación de una relación a largo plazo con sus clientes. (p.101)

Bose, (2012) lo define como: "La integración de tecnologías y los procesos de negocios usados para satisfacer las necesidades de los clientes durante cualquier interacción con los mismos" (p.13).

Finalmente Fernández \& Navarro, (2014). Define la gestión de las relaciones con el cliente como:

“La implementación o mejora de los procesos, organización y tecnología involucrados en la maximización del valor del cliente por medio de las interacciones de la empresa con éste a través de cualquier canal de comunicación". (p.15).

Sin duda que todas guardan una relación muy cercana en lo que respecta a la gestión o información que se tenga del cliente, y el valor que se le da, como clave del éxito para muchas empresas.

Sin embargo, hoy en día las estrategias de marketing han cambiado profundamente buscando encontrar 
esa ventaja competitiva que establezca un factor diferenciador perdurable en el tiempo; primeramente se estableció una orientación hacia la producción, donde la empresa no se preocupaba por las ventas, ya que las tenía aseguradas, y su actividad comercial se limitaba exclusivamente a mejorar el proceso productivo; seguidamente se pasó a una orientación al producto donde los consumidores preferían aquellos productos innovadores de mayor calidad; después saltó a una orientación a las ventas, donde se luchaba férreamente por el dominio del mercado, y los esfuerzos se centraban netamente en las ventas; y por último se da un gran paso hacia la orientación mercado donde ya las empresas tratan de conocer los gustos de los compradores potenciales para adaptar los productos a sus necesidades. Se empieza a dar muestras de lo que hoy se podría llamar como una orientación al cliente. En relación a esto, Lambin, Gallucci, \& Sicurello, (2009) describen en tre otras cosas que:

El objetivo del marketing relacional es desarrollar relaciones a largo plazo y mutuamente renbales no solo con los clientes sino con las multiples partes interesadas, aunque el foco de la administracion de las relaciones con el cliente (CRM) deberia ser primariamente el cliente. (p.104)

Se desarrollaron con el tiempo herramientas que pudieran gestionar información concreta de sus clientes para poderla aprovechar ofreciendo productos de forma más personalizada (Greenberg, 2008).

Aspectos muy relevantes como la rentabilidad del cliente, estilos y frecuencias de compra, tipos de productos demandados, y accesibilidad a los mismos, son algunas características en las que un sistema de información como el CRM plantea respuesta; al mismo tiempo como estrategia para almacenar toda información de forma tan detallada de los usuarios, de manera que pudiera ser aprovechada al máximo detalle y así establecer una ventaja competitiva.

\section{El proceso CRM}

Dado que el CRM es un proceso cíclico que involucra a diferentes áreas o departamentos de una organización, este proceso puede ser representado de varias formas; sin embargo, lo que se pretende es que se mejore continuamente por lo que se dice que este sistema de negocio es un proceso de mejora continua. (Fernández \& Navarro, H, 2014).

Esto involucra acciones tales como: conocer (análisis de clientes, análisis de competidores), seleccionar (nuevos productos, gestión de campañas), vender (contactos, gestión de clientes), servir (gestión post venta, quejas y reclamos). (Fernández \& Navarro, H, 2014. p. 119).

Sin embargo, el éxito en la adopción de este tipo de sistemas de información no está simplemente en su implantación, sino en analizar muy detalladamente las necesidades que tenga la empresa en función de las acciones comerciales concretas que se traduzcan en resultados positivos. (Dominguez, 2005).

El proceso de CRM inicia en la evaluación de clientes y así mismo establecer una clasificación de los mismos, de manera que se puedan obtener los mejores resultados en función de la información de cada uno de ellos.

Griffin (citado por Lambin, Gallucci, \& Sicurello, 2009) establece que:

Inicialmente se identifican los clientes sospechos, es decir aquellos potencial que puedan estar interesados en algun producto o servicio. Posteriormente se identifican los potenciales, lo cuales son los que tienen un interes mas fuerte en el producto o servicio y esten dispuestos a pagar por el. Despues estan los potenciales descalificados, quienes son rechazados puesto no tienen la rentabilidad que espera la empresa. (ver figura 6).

La empresa convertirá a los potenciales calificados en clientes de primerizos, y si están satisfechos en clientes o compradores repetidos. Por último y como reto final la empresa transformará a estos clientes en partidarios por lo que se presume que atraerán a otros clientes. (p.104) 
Figura 6.

\section{Tipología de clientes desde una perspectiva CRM}

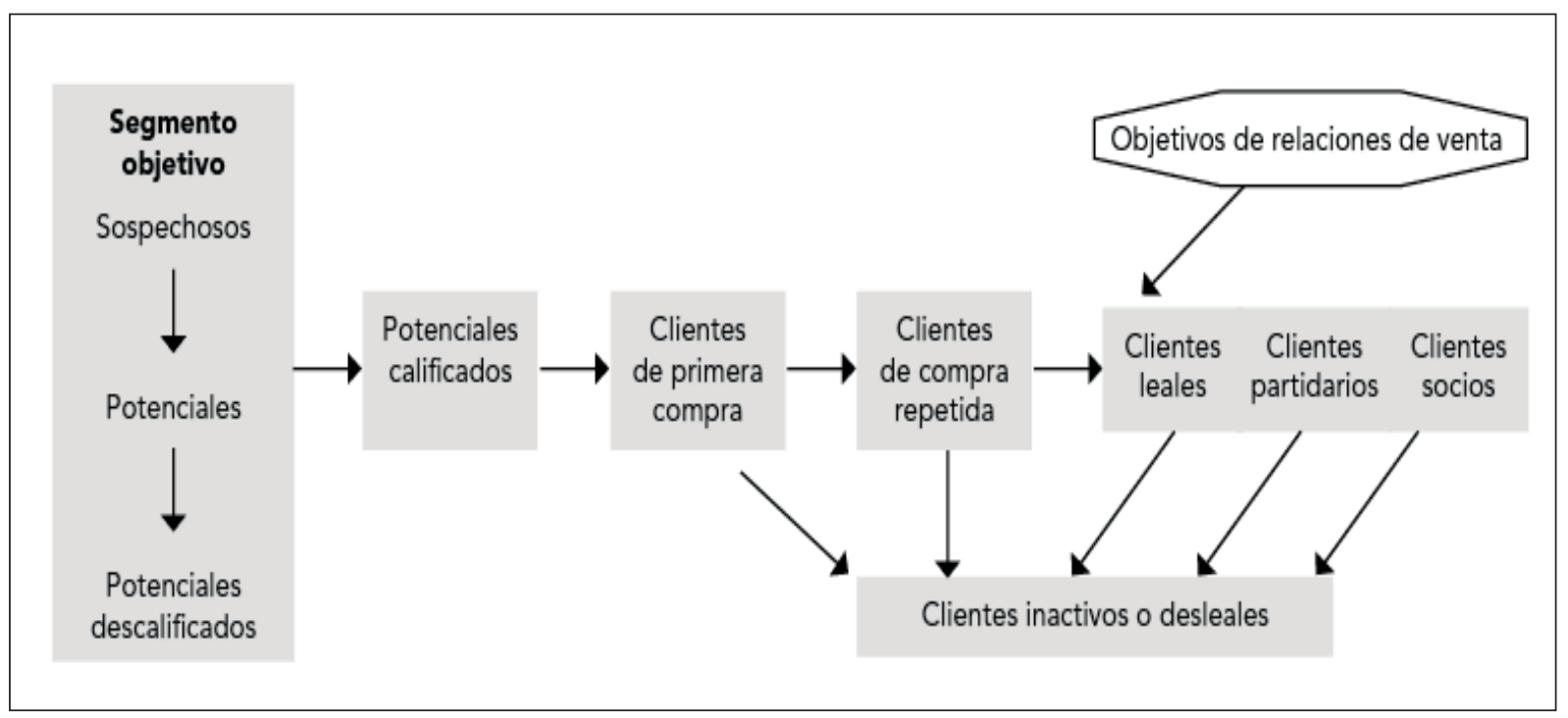

Fuente: Griffin (citado por Lambin, Gallucci, \& Sicurello, 2009, p. 104)

\section{Beneficios del CRM}

Los principales beneficios del CRM son: la mejora del servicio a clientes actuales, captación de nuevos clientes, y ayudar en la gestión de los departamentos de ventas, marketing y servicios (Sieber, Valor, \& Porta, 2006, p. 98).

Los beneficios de un CRM se traducen en un mayor conocimiento del cliente, en el aumento de la satisfacción y lealtad de los mismos, aumento de las ventas, y reducción de costes del servicio (Ayuso \& Rodríguez, A, 2011).

\section{Un caso de éxito de CRM: Amazon}

Jeff Bezos fue su fundador en 1994. Comenzó como una librería en línea que ofrecía libros en Estados Unidos y otros 45 países. Gracias a sus negocios virtuales pudo ofrecer 4 veces más de lo que una librería tradicional ofrecía por esos tiempos.

Amazon que revolucionó el mundo con su venta de libros y más tarde abrió sus fronteras a otros productos como la electrónica o el entretenimiento. Es una de las páginas que da más protagonismo al cliente, con numerosas referencias durante todo el proceso de consulta o compra de un producto que van desde la información sobre sus anteriores pedidos hasta la recomendación de otros productos relacionados con los que ha adquirido o por los que ha mostrado interés. Según la American Consumer Association, Amazon tuvo un $88 \%$ de clientes satisfechos con el servicio proporcionado, en los años 2002 y 2003, una cifra altísima para una empresa de servicios que no trata cara a cara con los clientes. (Sieber, Valor, \& Porta, 2006, p.87).

Amazon contempla su éxito estratégico en la adopción de los medios digitales, puesto ve en ellos un canal más efectivo para llegar a muchos más clientes de forma más rápida. Así mismo establece que por medio del mundo digital se podría ofrecer muchos más productos que en forma física, y es un hecho, puesto que en el mundo virtual las fronteras de las bodegas de almacenaje no existen metafóricamente hablando. 
Otra de las estrategias claves de éxito de Amazon consistía en emplear lo que llamamos teorías de long tail, larga cola. (Anderson, 2008) Por cuanto comercializaba libros pocos reconocidos, pocos especializados que podrán tener un sin número de clientes logrando así comercializar millones de unidades ya que habría productos para diversos segmentos para diversos estilos de clientes a cualquier precio; esto finalmente se traduciría en beneficios económicos para la empresa. Sin embargo, la cumbre del éxito llego cuando desarrolló el negocio referenciado como se mencionó inicialmente, llegando a más clientes haciendo compras solo por recomendación.

Arana, (2001) comenta en relación al CRM y a la estrategia comercial:

El eBusiness está transformando profundamente el entorno empresarial $y$, en particular, está produciendo una verdadera revolución en lo relativo a la gestión de las relaciones con clientes (Customer Relationship Management, CRM). En este sentido, PricewaterhouseCoopers Consulting ha verificado que más del $60 \%$ de los cambios que se generan en las empresas debido al eBusiness se relaciona con CRM (el resto de los cambios tiene que ver con otros aspectos de la gestión, como el eProcurement y la gestión de los recursos humanos). (p.1)

\section{Los sistemas Estratégicos}

El propósito de un sistema de información según Solares, Baca, \& Acosta, (2000) “estratégico es permitir a los altos ejecutivos tener acceso a los datos de interés, crear información útil y agrupar los resultados en una forma clara". (p.70).

\section{Ventajas}

Inclusive Solares, Baca, \& Acosta, (2000) contemplan las siguientes ventajas de los sistemas estratégicos:

- La disponibilidad de información relevante y confiable que representa un ahorro de tiempo para cada ejecutivo, quien quizá dedique más tiempo a la planeación estratégica del negocio que a la obtención de información.

- La empresa se asegura de que todos sus ejecutivos se enteren de la información más importante para la toma de decisiones.

- La implementación de los sistemas de información estratégica en las organizaciones hace posible la creación de ventajas competitivas para tomar decisiones más rápidas y acertadas que la competencia. (p.70).

Ahora bien, dentro de la clasificación de los sistemas estratégicos se destacan el EIS/BI, y el CMI o BSC; siendo este último el de más recordación y utilidad en la actual gestión de las empresas. Detallaremos los mencionados a continuación.

\section{El BSC (Balance Score Card)}

0 también conocido como cuadro de mando integral, y es una herramienta basada en indicadores estructurados en torno a las cuatro perspectivas claves de una organización (Kaplan \& Norton, 2000).

Estas perspectivas son: la financiera, de clientes, procesos internos, aprendizaje y crecimiento.

Con el BSC o CMI se pretende expandir mucho más la visión errada que se tiene de los indicadores de gestión, al ser solo los financieros los que más relevancia tienen, puesto que demuestran la utilidad y la rentabilidad de la empresa; sin embargo, el CIM ofrece el análisis de otros indicadores que son vitales para el futuro y el progreso de la compañía. Estos mismos son la clave en el éxito de la gestión empresarial, por medio de ellos se puede identificar cómo de cerca o de lejos se está de conseguir los objetivos planteados.

En relación con la estrategia de la alta dirección, los indicadores juegan un papel fundamental, puesto ayudan a medir cómo impacta dicha estrategia en todas las áreas de la organización; en la comunicación, en la planificación y fijación de objetivos, en la formación, y en la traducción de la visión corporativa.

Sin embargo, si desde la alta dirección no se clarifican dichos objetivos y la visión no está alineada 
estratégicamente con las operaciones de la empresa, el uso del BSC puede detectar estas falencias.

Algunos de los inconvenientes que trata de resolver el CMI o BSC son muy comunes en las empresas de hoy, por tanto, Martínez \& Milla, (2012) nos describe algunos muy tipicos:

- Estrategia no implementada en todos los niveles de la organización.

- Dificultad de entendimiento del lenguaje directivo por parte del resto de la organización.

- Visión a corto plazo.

- Excesiva concentración en indicadores financieros.

- Carencia de claridad en las relaciones causa-efecto lo que dificulta la toma de decisiones.

- Ausencia de nexo entre la estrategia de la empresa y la acción; lo que implica ausencia de resultados, logros y metas. (p. 195)

\section{El proceso de implantación del CMI}

Martínez \& Milla, (2012) en su libro de «cómo Implantar el cuadro de mando integral», estable una serie de fases a seguir para llevar a cabo este sistema estrategico: la planificacion, el proceso de reflexion estrategica, el desarrollo del mapa estrategico, implantacion, y por ultimo control y seguimiento (p.346).

También en el proceso implantación no se puede dejar de lado los componentes básicos que toda herramienta como ésta conlleva. Bustamante (2007) describe dichos componentes así:

- Mapa Estratégico (establece la estrategia de la organización).

- Matriz del CMI (guía para la acción y medición).

- Software ( visualizar resultados). ( p.12)

\section{Beneficios del BSC}

Dávila, (1999) nos presenta algunos de los beneficios de esta herramienta:

- La fuerza de explicitar un modelo de negocio y traducirlo en indicadores, facilita el consenso en toda la empresa, no sólo de la dirección, sino también de cómo alcanzarlo.
- Clarifica cómo las acciones del día a día afectan no sólo al corto plazo, sino también al largo plazo.

- Una vez el CMI está en marcha, se puede utilizar para comunicar los planes de la empresa, aunar los esfuerzos en una sola dirección y evitar la dispersión. En este caso, el CMI actúa como un sistema de control por excepción.

- También se puede utilizar como una herramienta para aprender acerca del negocio. En efecto, la comparación entre los planes y los resultados actuales ayuda al equipo de dirección a revaluar y ajustar tanto la estrategia como los planes de acción. (p.42).

El CMI parte de la estrategia planteada por la alta dirección, pero a su vez esta herramienta permite hacer un seguimiento intensivo a través de los indicadores dispuestos para evaluar dicha estrategia que puede ser traducida en objetivos precisos.

\section{El EIS (Executive information System)}

Cohen \& Asín, L, (2009) lo definen:

El EIS es un sistema computacional que provee al ejecutivo acceso fácil a la información interna y externa al negocio con el fin de dar seguimiento a los factores críticos para el éxito. Se enfocan primordialmente en proporcionar información de la situación actual de la compañía y dejan en un segundo plano la proyección de esta información hacia escenarios futuros.

Lapiedra, Davece, \& Guiral, (2011) describen el EIS:

Como un sistema de información computarizado concebido específicamente para su uso por parte de la alta dirección de la empresa, a quien le proporciona información tanto interna como externa que pueda utilizar de apoyo en el desempeño de sus tareas. (p.39). Concluyen asi mismo que el EIS se caractariza por brindar:

- Presentación de la información; permite ser adaptado en forma personalizada.

- Orientación a factores claves de éxito; es decir proporciona información de las variables claves del negocio. 
- Capacidad de comunicación y organización del tiempo; sirve de apoyo a las funciones de comunicación.

- facilidad de uso; en el sentido en que estos sistemas deben adecuarse al perfil del usuario. (p. 40)

\section{Claves de éxito de un EIS}

Al momento de decidirse por un sistema de información, se tiene la premisa que las expectativas de los resultados que brindará, serán muy altas, sin embargo, fracasan puesto no se tiene en cuenta ciertos aspectos fundamentales.

Algunos de estos factores a tener en cuenta al implementar un EIS según de Mazariegos, (2004) son:

- Acceso. Haciendo referencia a que los usuarios tendrán acceso a él, de una manera no complicada y desde puntos clave.

- Uso. Indica su frecuencia de uso. Si un sistema no es usado, o simplemente, los usuarios potenciales no lo emplean, esto se reflejará en el éxito del sistema.

- Satisfacción. Si el sistema no puede satisfacer a sus usuarios, éstos no usarán el sistema.

- Impacto Positivo. Un sistema es exitoso si tiene un impacto benéfico en los ejecutivos y la organización.

- Difusión. Otro punto que indica el éxito es la propagación del sistema. (p. 4,5)

En definitiva, el EIS es una herramienta tecnológica muy útil, que brinda a la alta dirección comprender el entorno interno y externo de la organización y las acciones que participan en el para así establecer oportunas decisiones llegado el caso.

\section{Conclusión}

El factor tecnológico está influyendo de manera definitiva en la forma como hacer los negocios. También como está produciendo importantes cambios en las impresiones de los consumidores y como las empresas deben responder a estos cambios. De igual forma las ricas experiencias que los clientes tienen en el consumo de determinados productos, están provocando que las expectativas de estos se incrementen a la hora de decidirse por cualquier producto o servicio. En este sentido, ha cobrado vital importancia el desarrollo de los sistemas de información, las comunicaciones y los canales por donde estas se transmiten. Es así que la inteligencia de los negocios se convierte una herramienta fundamental en la dirección estratégica.

En primera instancia se consideran procesos de negocio dentro de una empresa, a cada una de las actividades relacionadas entre sí para dar solución a una necesidad propia de la organización. Estos procesos de negocios, aunque existen muchos, pueden clasificarse en dos grupos: los procesos o sistemas transaccionales y los sistemas estratégicos.

Los llamados sistemas transaccionales administran la información al máximo detalle posible y representan hoy la solución para la gestión de las operaciones en las empresas. La planificación de los recursos de la organización o (ERP), proporcionan eficiencia al interior de la compañía sistematizando y centralizando sus procesos. La gestión de la cadena de suministros o (SCM) se encarga de trasladar dicha eficiencia a las relaciones con los proveedores. Pero también están los sistemas de administración de relaciones con los clientes o (CRM), quizás el de más recordación; dado que surge de las necesidades que tienen las empresas de conocer mejor a sus clientes y de también de desarrollar tecnologías que gestionen grandes volúmenes de datos. Este sistema de información analiza a los clientes a través de su comportamiento para adaptar la propuesta de valor a cada uno de ellos.

Los tres procesos anteriores tienen como objetivo contribuir a la toma de decisiones de la empresa y aportar estabilidad a la hora de integrar nuevos sistemas y procesos.

Por otra parte, se encuentran los sistemas estratégicos, estos sumarizan la información de la empresa alineando las acciones con la estrategia empresarial propuesta en el mediano- largo plazo. Dentro de los más reconocidos y familiarizados con el entorno corporativo se encuentra el cuadro de mando integral (CMI) o el Balance Score Card (BSC), el cual proporciona una visión de los principales indicadores de gestión de la compañía enmarcados 
en cuatro grandes dimensiones o perspectivas: la financiera, la de los clientes, la de los procesos, y la de las personas. Hoy en día se habla inclusive de una quinta perspectiva crucial en el éxito de la gestión corporativa, y es la responsabilidad social empresarial.

Se encuentra por último el sistema de información para ejecutivos (EIS) que, aunque no es tan familiar como el BSC, permite analizar la información actual de la empresa sin contempla proyecciones futuras.

Ambos sistemas tienen una principal característica en común, son utilizados por la alta dirección, puesto que los ayuda a la toma de decisiones y mirar desde una visión más amplia el funcionamiento de la organización en general.

Finalmente, el impacto que ha tenido la implementación de los sistemas de información en las empresas, ha sido muy favorable; casos representativos a nivel global como lo es Amazon, Bimbo, Netflix, en la cuales han significado la clave del éxito en la gestión empresarial, dan fe y testimonio que los ambientes tecnológicos pueden llegar a ser el canal más favorable si se hace un buen análisis de la situación actual de empresa en función de poder canalizar los procesos de negocio. No obstante, aún queda un largo camino por recorrer, estando en plena era de la información, existe la necesidad que las compañías evalúen muy bien sus necesidades con el fin de poder contemplar la adopción de algún sistema de información que la consolide y le brinde la estabilidad que tanto ambicionan.

Se viene creciendo a pasos agigantados; para el caso de Latinoamérica con la inclusión de grandes compañías que implementan sistemas de información, en especial CRM, cobra más importancia el conocimiento del mercado en función de que las marcas busquen satisfacer las necesidades de sus clientes.

Para Colombia y Latinoamérica en general existen fuertes tendencias de crecimiento en la implementación de por lo menos un sistema de información; sin embargo, aún existe la debilidad en la complementación de tecnologías particularmente en el campo de aplicaciones que se perfeccionan con estos procesos de negocio. En este sentido se recomienda para fu- turas investigaciones plantear estudios que midan y categoricen empresas del sector, que estén en la necesidad de adoptar algún sistema de negocio de acuerdo a una evaluación sistemática y concisa de su situación que las pueda catapultar a una situación favorable en un mercado cada vez más competitivo.

\section{Referencias Bibliográficas}

Anderson, C. (2008). The Long Tail, Whay the future of business Is Selling less of More.

Arana, A. (2001). El impacto de internet en la transformacion de las relaciones con los clientes (CRM). Harvard Deusto. Marketing \& Ventas, 70.

Arjonilla, D., \& Medina, G, J. A. (2013). La gestión de los sistemas de información en la empresa. Teoría y casos prácticos. Ediciones Pirámide.

Ayuso, S., \& Rodríguez, A, V. M. (2011). manual soluciones CRM. Formación empleo. Editorial CEP.

Berenguer, J. M., \& Ramos-Izquierdo, J. A. (2003). Negocios digitales: competir usando tecnologias de información. EUNSA.

Bose, R. (2002). Customer relationship management: key components for IT success.

Bustamante, L. A. (febrero de 2007). Estrategia empresarial la administración estratégica y el cuadro de mando integral. Intercambio Técnico ATDL, 12,13. Obtenido de www. atdl.org.

Cano, J. (2007). Business Intelligence: competir con información.

Cavinato, J. L. (1991). Identifying interfirm total cost advantages for supply chain competitiveness.

Chavez, J. H. (2000). Supply Chain Management (2a. ed.). RIL editores.

Christopher, M. (1998). Logistics and Supply Chain Management. London.

Cobarsí, M. (2011). Sistemas de información en la empresa. Editorial UOC.

Cohen, K. D., \& Asín, L. E. (2014). Tecnologias de la información (6a. ed.). Mexico D.F: McGraw-Hill Interamericana.

Cohen, K., \& Asín, L, E. (2009). Tecnologias de informacion en los negocios. Mc Graw Hill.

Cooper, M. C., \& Ellram, L. M. (1993). Characteristics of supply chain management and the implications for purchasing and logistics strategy.

Davenport, T. H., \& Prusak, L. (1999). Working Knowledge: How Organizations Manage What They Know.

Davenport, T., \& Prusak, L. (1997). Ecología de la Información. Dávila, A. (1999). Nuevas herramientas de control: El cuadro de mando integral. REVISTA DE ANTIGUOS ALUMNOS. IESE, 7. 
Helmer Muñoz-Hernández, Roberto Carlos Osorio-Mass y Luis Manuel Zúñiga-Pérez

Dávila, L. F. (2005). Hacia la inteligencia del negocio con Excel 2003. Editorial Politécnico Grancolombiano.

De Mazariegos, A. (9 de Mayo de 2004). Sistemas de información para ejecutivos. Obtenido de gestiopolis: http://www. gestiopolis.com/sistemas-de-informacion-para-ejecutivos/

Dominguez, J. I. (17 de Marzo de 2005). Visiones de CRM. Obtenido de Gestiopolis: http://www.gestiopolis. com/4-visiones-crm/

Fernández, 0. M., \& Navarro, H. M. (2014a). Sistemas de Gestión Integradas para las Empresas (ERP). Alcalá Henares España: Servicio de Publicaciones. Universidad de Alcalá.

Fernández, 0., \& Navarro, H, M. A. (2014b). Sistemas de Gestión de Relaciones con Clientes en las Empresas (CRM). UAH. Servicios de publicaciones.

Gattorna, J. (2011). Cadenas de abstecimiento dinámicas: como movilizar la empresa alrrededor de lo que los clientes quieren. Ecoe. Ediciones.

Greenberg, P. (2008). las Claves del CRM: Gestión de las relaciones con el cliente. Mac Graw Hill.

Herrera, N. (2015). Sistemas de inteligencia de negocios. Obtenido de Gestiopolis: http://www.gestiopolis.com/ sistemas-de-inteligencia-de-negocios/

Howson, C. (2010). Business Intelligence: Estrategias para una implementación exitosa. McGraw-Hill Interamericana.

Kaplan, R. S., \& Norton, D. P. (2000). Cómo utilizar el cuadro de mando integral: para implantar y gestionar su estrategia.

Lambert, D. M., \& Cooper, M. C. (2000). Issues in Supply Chain Management, Industrial Marketing Management.

Lambin, J., Gallucci, C., \& Sicurello, C. (2009). Dirección de marketing: gestión estrategica y operativa del mercado (2a. ed.). Mexico D.F: McGraw-Hill Interamericana.

Lapiedra, A. R., Davece, C. C., \& Guiral, H. J. (2011). Introducción a la gestion de sistemas de información en la empresa. España: Universitat Jaume I. Servei de Comunicació i Publicacions.

Marco, S. J., \& Marco, G. M. (2013). Sistemas de información (en las organizaciones). España: UOC.

Martínez, P. D., \& Milla , G. A. (2012a). Cómo Implantar el cuadro de mando integral. Madrid. España: Ediciones Diaz de Santos.

Martínez, P. D., \& Milla, G. A. (2012b). Introducción al cuadro de mando integral. Madrid. España: Ediciones Diaz de Santos.

Médes, d. R. (2006). Más allá del Business Intelligence. 16 experiencias de exito.
Mozqueda, L. (2016). Desafios de implementar un ERP. Obtenido de Grades Pymes: http://www.grandespymes. com.ar/2016/03/11/desafios-de-implementar-un-erp/

0 'Brien, J. A. (2006). Sistemas de información gerencial (7a. ed.). Mexico D.F: McGraw-Hill Interamericana.

Pires, S., \& Carretero, D, L. E. (2007). Gestión de la cadena de suministro. España: Mc Graw Hill.

Polo, A, A. M. (9 de Mayo de 2009). Definición y Herramientas de la Inteligencia de Negocios. Obtenido de Gestiopolis: http://www.gestiopolis.com/ definicion-herramientas-la-inteligencia-negocios/

Portal, R. (2011). Supply Chain Management (SCM): Gestión Logística Integral Aplicada. Obtenido de Gestiopolis: http://www.gestiopolis.com/supply-chain-managementscm-gestion-logistica-integral-aplicada/

Rocha, L. M. (1 de Marzo de 2007). Sistemas de información. Obtenido de Gestiopolis: http://www.gestiopolis.com/ sistemas-de-informacion/

Rodriguez, P. (2014). Cómo hacer inteligente su negocio: business intelligence a su alcance. Grupo Editorial Patria.

Rojas, G, K. Y. (3 de Mayo de 2016). Inteligencia en los negocios. Base de datos y CMI. Obtenido de Gestiopolis: http://www.gestiopolis.com/inteligencia-los-negocios-bases-datos-cmi/

Sánchez, S. F., Toharia, R. J., \& Raya, G. L. (2000). Lenguajes de marcas y sistemas de gestion de la información. RA-MA.

Sieber, S., Valor, J., \& Porta, V. (2006). Los sistemas de información en la empresa actual. Aspectos estratégicos y alternativas tácticas. Mc Graw Hill.

Solares, S. P., Baca, U. G., \& Acosta, G. E. (2000). Administracón informática: análisi y evaluacion de tecnologias de la información. Larausse- Grupo Editorial Patria.

Sosa, F. M., \& Hernández, P. F. (2007). Arquitectura de sistemas informativos para la toma de decisiones. Cordoba. Argentina: El cid Editor.

Velásquez, J. (2013). Caso Bimbo. Obtenido de Academia: http://www.academia.edu/8060654/CASO_BIMBO

Velazco, S. J. (2013). Gestión de la logistica en la empresa: Planificación de la cadena de suministros. Madrid. España: Larausse-Ediciones Pirámide.

Zarate, G. (2013). Inteligencia de negocios. Obtenido de Gestiopolis: http://www.gestiopolis.com/ inteligencia-de-negocios/ 